\title{
Research on application method of uncertainty quantification technology in equipment test identification
}

\author{
Jiajia Wang ${ }^{1,2, *}$, Hao Chen ${ }^{1,2}$, Jing $\mathrm{Ma}^{1,2}$, and Tong Zhang ${ }^{1,2}$ \\ ${ }^{1}$ Beijing Jinghang Research Institute of Computing and Communication Beijing, China \\ ${ }^{2}$ Applied Mathematics Research Center of China Aerospace Science and Industry Group Beijing, \\ China
}

\begin{abstract}
This paper introduces the concepts of equipment test qualification and uncertainty quantification, and the analysis framework and process of equipment test uncertainty quantification. It analyzes the data uncertainty, model uncertainty and environmental uncertainty, and studies the corresponding uncertainty quantification theory to provide technical reference for the application of uncertainty quantification technology in the field of test identification.
\end{abstract}

\section{Introduction}

With the development of weapon equipment, the demands are more large-scale, complex and precision, the comprehensive test of the system has the characteristics of high cost, long cycle and many restrictions by objective conditions. Moreover, most of the whole machine test is not repeatable, the test data that can be obtained is also very limited. At the same time, due to the limitation of understanding the physical mechanism of the system, it is difficult to guarantee the authenticity and accuracy of the model in the virtual test, which is a challenging problem for the overall evaluation of the system. In order to reduce the development time and cost of aerospace system and equipment as much as possible, explore the test methods and means that can reflect the combination of virtual and real system, quantify the impact of uncertainty factors, and carry out the research on quantitative technology of equipment test uncertainty, which can improve the test efficiency, shorten the development cycle, reduce the test cost and ensure the quality of equipment ${ }^{[1]}$.

In recent years, the processing methods for specific uncertainty problems have been proposed and developed. DARPA plans to carry out a new mathematical research project "quantifying the uncertainty of physical systems". The project aims to solve this problem by developing mathematical tools and methods, which can effectively quantify and manage multi-source uncertainty, thus replacing expensive repetitive experiments. This paper studies the characteristics of equipment that affect test design, test implementation and test evaluation in the process of different types and stages of test, defines the uncertainty factors

* Corresponding author: gaga214@,216.com 
of various stages and types of tests, and analyzes the application approaches of uncertainty quantification technology combined with the theory and method of uncertainty quantification technology, so as to provide reference for its application.

\section{Uncertainty quantification technology}

Uncertainty can be divided into two categories: Aleatory Uncertainty and Empirical Uncertainty. The former exists in nature, and this kind of uncertainty can't be eliminated. It can be analyzed by probability theory, also known as objective uncertainty. The latter is mainly due to the lack of knowledge, lack of data, cognitive bias and so on. It can be eliminated by improving data and improving cognition through in-depth learning, which is also called subjective uncertainty.

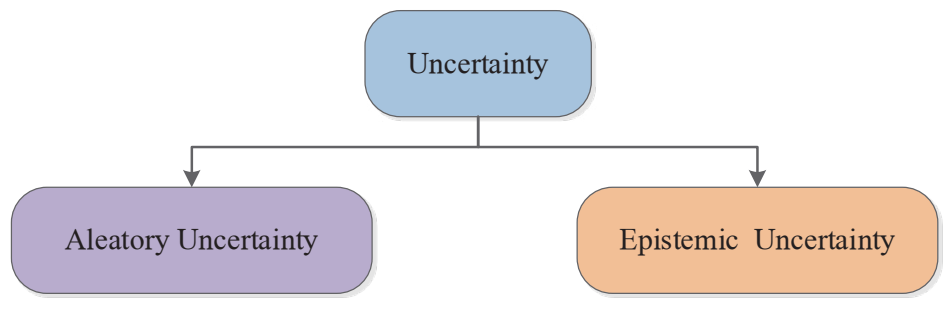

Fig. 1. Uncertainty classification.

There are two kinds of uncertainty quantification methods in engineering problems: probability method and non-probability method. Among them, non-probabilistic methods include fuzzy sets theory, D-S evidence theory, possibility theory, convex model and so on.

\subsection{Probability method}

Probability method is a widely accepted method to quantify uncertainty by using random variables to represent uncertainty. In specific engineering applications, according to the sample information, a variety of uncertainties can be expressed in the form of probability density function, such as the prior information of measurement data and model parameters, and the uncertainty parameters related to input and output. The basic process of this method is as follows: firstly, the probability distribution model is assumed according to the actual situation, then the probability distribution function of model parameters is fitted by mathematical statistics method, and finally the distribution probability of variables in a certain interval is determined ${ }^{[2]}$. The commonly used probability methods are shown in the Fig. 2.

It has a perfect theoretical basis to quantify uncertainty by using probability method, which can accurately and easily deduce the uncertainty quantification and transfer. However, when applied to practical engineering problems, the method also has some limitations. For example, in order to obtain the probability density function, certain sample information is needed to support the method. In addition, the method relies on the amount of sample information When there are multiple variables, it is still difficult to accurately express the joint probability density after the probability density of a single variable is obtained. 


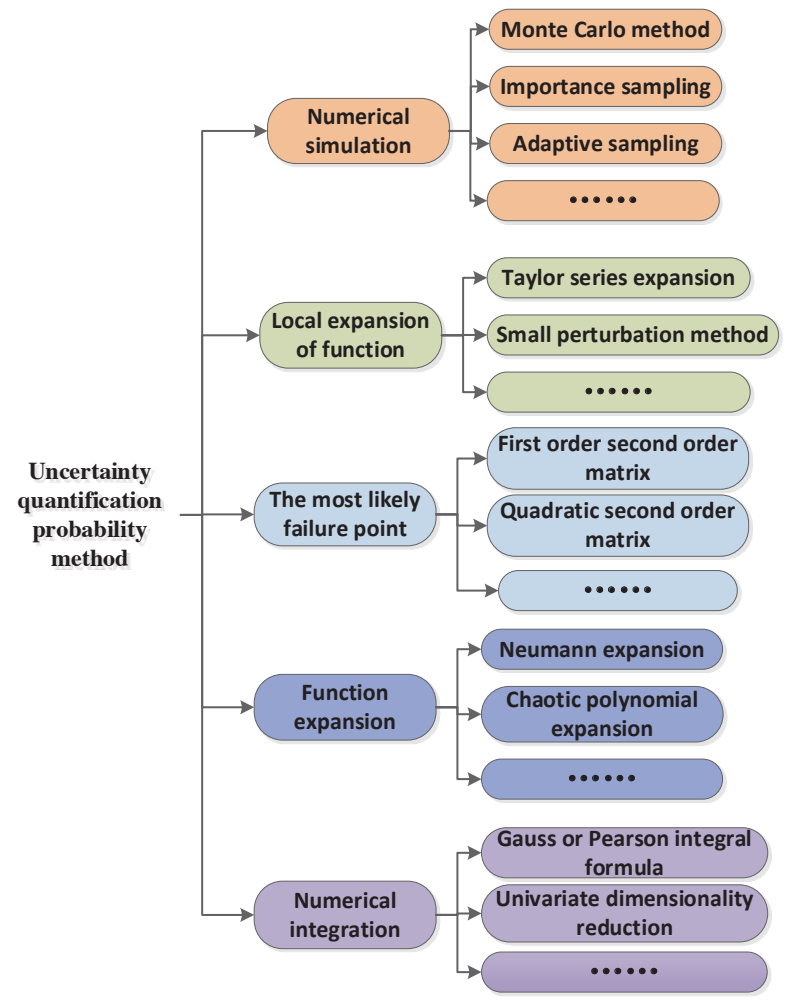

Fig. 2. Probabilistic classification.

\subsection{Non-probabilistic method}

Traditional probability methods have many limitations in dealing with cognitive uncertainty. Therefore, non-probabilistic uncertainty quantification methods, such as evidence theory ${ }^{[5]}$, fuzzy set theory, interval theory, possibility theory and convex set theory, are proposed and used to solve the reliability evaluation of complex systems under cognitive uncertainty.

Evidence theory uses quality function, trust function and likelihood function to characterize and quantify cognitive uncertainty; fuzzy theory describes the discrete state of parameters by membership function; interval theory describes the change of parameters by defining the range of parameter change; possibility theory is mostly used to quantify uncertainty modelling when there is less information, compared with probability distribution function in probability method Convex set model is a non-probabilistic modelling method which uses convex set theory to describe uncertainty. It needs less detailed information and can fully reflect the uncertainty problem, so it has been paid attention in many fields of engineering problems. The specific methods are as Fig. $\mathbf{3}$.

\section{Research on uncertainty quantification of equipment Identification}

Considering the connotation of information equipment test, uncertainty analysis is carried out for equipment test evaluation performance test, operational test and in-service assessment test. Equipment test uncertainty mainly comes from data uncertainty, model uncertainty and environmental uncertainty. Model uncertainty is the uncertainty of model 
expression, model selection and model parameters. Among them, the model parameter uncertainty is usually the interval description given by experts, or the simple probability distribution with little information; the data uncertainty is usually reflected in the process of data acquisition and observation, the uncertainty of data sampling, and the uncertainty of data rules; the environmental uncertainty includes complex electromagnetic environment, complex geographical environment, complex meteorological environment and so on It is similar to the uncertainty of actual combat environment and equipment storage environment.

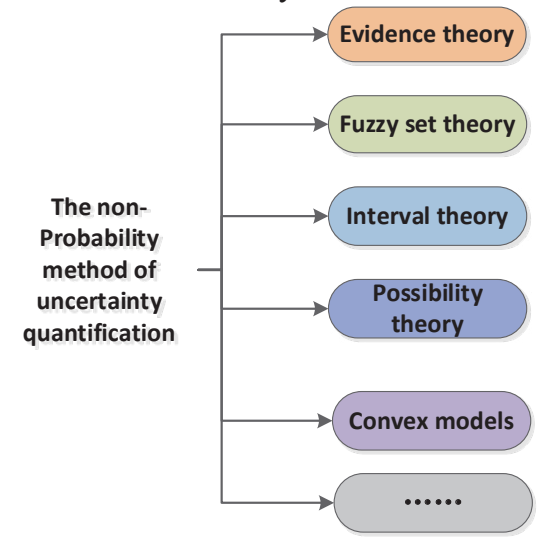

Fig. 3. Non-probabilistic classification.

In the process of equipment test, it is divided into virtual test, hardware in the loop simulation test and physical test according to the test environment. After the influence of uncertainty factors of various tests is analyzed, the corresponding calculation model is established, including the model itself, input variables, external disturbance and other uncertainties, so that the design and evaluation of the test depend on the number of tests in the test stage of the calculation model According to the difficulty of processing, the study aimed at the uncertainty in the calculation model of this type of engineering system, and provided a method reference for the overall evaluation of the system ${ }^{[3]}$

Considering the equipment test, the system modelling is not perfect due to the lack of understanding of the system characteristics, initial state and surrounding environment, and the model calculation is not accurate due to program errors, numerical errors and numerical approximation in the algorithm. In addition, the model parameters cannot be calibrated due to insufficient test data or large error, which also leads to the uncertainty of calculation model.

Because the multi-source uncertainty seriously affects the prediction accuracy of the calculation model to the real system, the comprehensive uncertainty analysis is very important for the experimental design, evaluation and operation decision-making of the engineering system. Uncertainty quantification is to identify, describe and analyze all the factors that affect the accuracy of the calculation model, including the expression of uncertainty, the study of the propagation of uncertainty, and the identification of model parameters and model bias through test data ${ }^{[4]}$. The research approach of uncertainty quantification technology is shown in Fig. 4. 


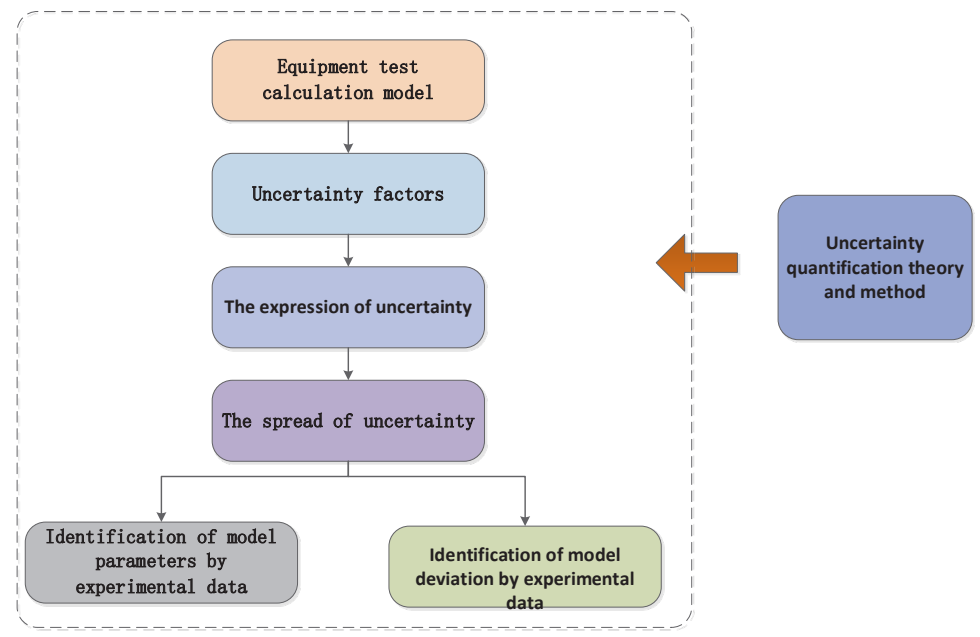

Fig. 4. Research approach of uncertain quantification technology.

\section{Conclusions}

In view of the new problems and challenges faced by equipment system test and evaluation, there are many uncertain factors in the evaluation process of equipment system, which are difficult to quantify. Based on the characteristics of equipment test and evaluation, combined with the theory and method of uncertainty quantification technology, this paper explores the application framework and approach of equipment test uncertainty quantification technology. In practical application, we should fully consider the uncertainty of equipment test for the pertinence and limitation of deterministic quantification method, the following research will deeply explore the mathematical model and algorithm of uncertainty quantification, and provide more scientific theoretical basis for the application of equipment test identification.

\section{References}

1. Crespo L G, Kenny S P, Giesy D P. The NASA Langley multidisciplinary uncertainty quantification challenge[C].16th AIAA Non-Deterministic Approaches Conference. 2014: 1347.

2. Roy C J, Oberkampf W L. A comprehensive framework for verification, validation, and uncertainty quantification in scientific computing [J]. Computer methods in applied mechanics and engineering, 2011, 200(25-28): 2131-2144.

3. Operational Test \& Evaluation Manual [R]. Marine Corps Operational Test and Evaluation Activity, 2011.

4. Profir B, Eres M H, Scanlan J, et al. Uncertainty Quantification via Elicitation of Expert Judgements[C].16th AIAA Aviation Technology, Integration, and Operations Conference. 2016: 3459.

5. Mourelatos Z P, Zhou J. A design optimization method using evidence theory [J]. Journal of mechanical design, 2006, 128(4): 901-908. 